\title{
Less is more: role of additional chemotherapy to concurrent chemoradiotherapy in locoregionally advanced nasopharyngeal cancer management
}

\author{
Yong Chan Ahn \\ Department of Radiation Oncology, Samsung Medical Center, Sungkyunkwan University School of Medicine, Seoul, Korea
}

Concurrent chemoradiation therapy (CCRT) has played the most important and central role in the definitive therapy for the patients with locoregionally advanced stage nasopharynx cancer. The addition of induction chemotherapy (IC) or adjuvant chemotherapy (AC) to CCRT have been widely accepted with the rationale of improving distant control in the clinical practices. This review article investigated the role of IC and AC based on 11 recent meta-analysis publications, and found that the clinical benefits obtained by the additional IC or AC to CCRT, at the cost of the increased risks of more frequent and more severe side effects, seemed not big enough. More intervention is not always better, however, less seems frequently good enough. The author would speculate that 'less is more' and would advocate CCRT alone as the current standard.

Keywords: Chemotherapy, Radiation therapy, Nasopharynx cancer

\section{Introduction}

Radiation therapy (RT) has played the most important and central role in the definitive therapy for the patients with locoregionally advanced and non-metastatic stage nasopharynx cancer. Except at its earliest stage, the addition of systemic chemotherapy to RT has been typically recommended with the proven benefits of improving the clinical outcomes. There are three main ways of combining systemic chemotherapy and RT with respect to the time sequences of each modality. The classic combination ways include the induction (or neoadjuvant) chemotherapy (IC) before definitive RT and the adjuvant chemotherapy (AC) following definitive RT. The third combination way, which is most recent and most popularized nowadays, is the concurrent delivery of chemotherapy during the RT course (CCRT). The most important theoretical rationale of adding either IC or $A C$ to definitive local RT is to help control probable distant micrometastasis, which often exists outside the local RT reach. On the other hand, synergy is the most important and powerful rationale of CCRT, which can usually enhance local tumor control with or without improving control of distant micrometastasis. In addition to these three classic ways, however, there are total of eight variations of combining RT and chemotherapy, some of which have been employed in the real-world clinical practice setting (Fig. 1).

There have been many comparative studies to investigate the roles of the additional IC or AC to RT alone or CCRT

Received 03 June 2019, Revised 21 June 2019, Accepted 24 June 2019.

Correspondence: Yong Chan Ahn, Department of Radiation Oncology, Samsung Medical Center, Sungkyunkwan University School of Medicine, 81 Irwon-ro, Gangnam-gu, Seoul 06351, Korea. Tel: +82-2-3410-2602, E-mail: ahnyc@skku.edu (https:// orcid.org/0000-0002-1971-8472)

(c) This is an Open Access article distributed under the terms of the Creative Commons Attribution Non-Commercial License (http://creativecommons.org/ licenses/by-nc/4.0/) which permits unrestricted non-commercial use, distribution, and reproduction in any medium, provided the original work is properly cited.

www.e-roj.org 


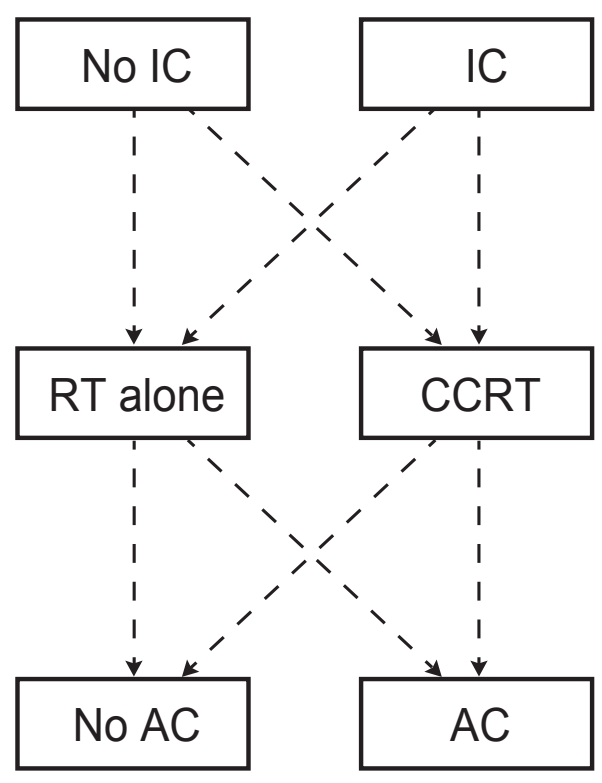

Fig. 1. There are theoretically 8 ways of combining radiation therapy and systemic chemotherapy. IC, induction chemotherapy; $\mathrm{RT}$, radiation therapy; CCRT, concurrent chemoradiation therapy; $A C$, adjuvant chemotherapy.

settings, typically through the phase 3 randomized controlled trial (RCT). And, based on these trials, several important metaanalysis reports have been published recently [1-11]. The most recent guideline by the National Comprehensive Cancer Network (NCCN) on the treatment of stage II-IVb nasopharynx cancer highly recommends the clinical trials, and listed two options of IC + CCRT and CCRT + AC as category 2a, while CCRT alone as category $2 b$, respectively [12]. This article is to review and summarize the purposes, the main results, and the key conclusions of 11 recent meta-analysis publications, and to see whether the NCCN guideline is reasonable. Practically all the meta-analysis publications were based on multiple RCTs, and 4 were in meta-analysis format, 5 were in network metaanalyses (NMA) format, and 2 were in individual patient data (IPD) pooled meta-analysis format, respectively. Among these, 5,1 , and 5 publications' main scopes were to investigate the role of $I C, A C$, and both, respectively (Table 1).

\section{CCRT versus RT Alone}

The greatest survival benefit, mainly by virtue of synergy effect, has been achieved by CCRT approach in many cancer types including nasopharynx cancer, when compared with the IC or AC approaches. During the RT course, by concurrent administering single agent (typically cisplatin) at lower dose intensity than that used in sequential administration, the toxicity profiles have become well tolerated by most patients with the improved cytotoxic effects that can be translated into better clinical outcomes. Based on this, CCRT has become the current standard in treating the patients with many cancer types including locoregionally advanced stage nasopharynx cancer. This superiority of CCRT over RT has been re-confirmed by a few meta-analysis reports listed in Table 1 , whose main scope was to investigate the role of IC or AC but partly compared RT alone and CCRT. The update of the meta-analysis of chemotherapy in nasopharynx carcinoma (MAC-NPC) metaanalysis by Blanchard et al. [1] confirmed and endorsed that CCRT, when compared with RT alone significantly improved overall survival (OS).

\section{Publications Mainly Focused on Role of IC}

There were 5 publications that mainly investigated the role of IC in addition to CCRT: 3 meta-analysis, 1 NMA, and 1 IPD meta-analysis, respectively. The most consistently reported clinical benefit by the addition of IC was the improved distant control (DC) or delaying distant metastasis. The benefit of overall survival (OS), however, was demonstrated by 3 publications, but not by the other two. Song et al. [2] compared the long-term efficacy of IC in addition to CCRT by meta-analysis of 4 RCTs including 798 patients. They documented that the benefit by adding IC was improved progression-free survival (PFS) and DC, which, however, were neither translated into improved OS nor locoregional control (LRC). Tan et al. [3] did systematic review and meta-analysis to investigate the role of IC in CCRT setting. Based on 6 RCTs and 5 observational studies, they could accrued 2,802 patients and found that the additional IC significantly improved PFS and OS, but also at the cost of more frequent acute side effects. Meta-analysis by Want et al. [4] assessed the role of IC in CCRT setting by collecting 9 RCTs that included 2,215 patients, where 7 trials compared IC + CCRT and CCRT, and 2 did IC + CCRT and CCRT + AC, respectively. They observed the addition of IC significantly improved OS and DC, but not LRC. These benefits, however, were achievable at the cost of significantly increased risk of grade 3-4 hematologic toxicities. Chen et al. [5] assessed the role of IC in addition to CCRT by meta-analysis on 9 RCTs that included 1,988 patients: 4 trials compared IC + RT and RT, 3 did CCRT and RT, and 2 did IC + CCRT and CCRT, respectively. They observed significantly improved DC by the addition of IC to CCRT when compared with CCRT alone, but there was no OS difference, which could have been due to 
Table 1. List of recent meta-analysis publications

\begin{tabular}{|c|c|c|c|c|}
\hline Study & Method & $\begin{array}{l}\text { Trials (no. of } \\
\text { patients) }\end{array}$ & Main scope & Key conclusions \\
\hline Song et al. [2], 2015 & MA & $4(798)$ & Role of IC & $\begin{array}{l}\text { IC improved DC. } \\
\text { No benefit in OS and LRC. }\end{array}$ \\
\hline Tan et al. [3], 2018 & MA & $11(2,802)$ & Role of IC & IC+CCRT improved OS and delayed progression. \\
\hline OuYang et al. [8], 2013 & MA & $11(2,605)$ & Role of IC & $\begin{array}{l}\text { IC improved OS and LRC. } \\
\text { AC improved DC. }\end{array}$ \\
\hline Wang et al. [4], 2016 & MA & $9(2,215)$ & Role of IC & IC improved PFS and OS. \\
\hline Chen et al. [5], 2015 & NMA & $9(1,988)$ & Role of IC & $\begin{array}{l}\text { IC improved DC. } \\
\text { No benefit in OS and LRC. }\end{array}$ \\
\hline Chen et al. [7], 2015 & NMA & $8(2,144)$ & Role of AC & $\begin{array}{l}\text { CCRT+/-AC improved OS and DC compared with RT. } \\
\text { No difference between CCRT+AC and CCRT. }\end{array}$ \\
\hline Yu et al. [9], 2016 & NMA & $11(2,626)$ & Both & $\begin{array}{l}\text { No difference among CCRT, IC+CCRT, and CCRT+AC. } \\
\text { IC+CCRT marginally improved DC compared with CCRT. }\end{array}$ \\
\hline Ribassin-Majed et al. [11], 2017 & NMA & $20(5,144)$ & Both & $\begin{array}{l}\text { AC+CCRT achieved highest OS. } \\
\text { IC+CCRT achieved highest DC. }\end{array}$ \\
\hline Liu et al. [10], 2018 & NMA & $12(3,248)$ & Both & $\begin{array}{l}\text { IC+CCRT improved OS, DC, and LRC. } \\
\text { No difference between IC+CCRT and CCRT+AC or between } \\
\text { CCRT+AC and CCRT. } \\
\text { RT alone is worst. }\end{array}$ \\
\hline Chen et al. [6], 2018 & IPD MA & $4(1,193)$ & Both & IC+CCRT improved OS by DC. \\
\hline Blanchard et al. [1], 2015 & IPD MA & $19(4,806)$ & Both & CCRT improved OS. \\
\hline
\end{tabular}

MA, meta-analysis; NMA, network meta-analysis; IPD, individual patients' data; IC, induction chemotherapy; $A C$, adjuvant chemotherapy; CCRT, concurrent chemoradiation therapy; RT, radiation therapy; DC, distant control; OS, overall survival; LRC, locoregional control; PFS, progression-free survival.

significant worse LRC. Chen et al. [6] did IPD pooled metaanalysis, based on 4 RCTs, all from the endemic region, in order to assess the role of IC + CCRT. They accrued 1,193 patients, all of who patients were treated with intensity-modulated RT technique and concurrent with cisplatin during the RT course. They demonstrated the significant improvements in PFS and OS, mainly associated with improved DC, by adding IC to CCRT when compared with CCRT alone. This publication has the merit of quite homogenous RT technique and dose schedules, all of which were performed at the high-volume centers within the endemic regions of nasopharynx cancer.

Based on these publications whose main scope was to investigate the role of $I C$, the improvement in $D C$ seemed rather consistently reported, while the OS benefit was not.

\section{Publication Mainly Focused on Role of AC}

There was 1 NMA publication that mainly investigated the role of AC in addition to CCRT. Chen et al. [7] did Bayesian NMA by collecting 8 RCTs that included 2,144 patients. Among these 8 trials, 5 compared CCRT + AC and RT, 1 did CCRT +
AC and CCRT, and 2 did CCRT and RT. The clinical outcomes following CCRT + AC and CCRT were significantly better than those following RT alone. However, there was no significant difference of the outcomes between CCRT + AC and CCRT. The authors suggested reduced toxicity by omitting AC.

\section{Publications with Multiple Comparisons Including Both IC and AC}

There were 5 publications that tried multiple comparisons: 1 meta-analysis, $3 \mathrm{NMA}$, and 1 IPD meta-analysis, respectively. The key information from these 5 publications was not consistent. OuYang et al. [8] did meta-analysis in order to investigate the role of IC and AC. They extracted 6 RCTs that compared RT or CCRT with or without IC and 5 RCTs that compared RT or CCRT with or without AC. They observed absolute 3 -year OS benefit of $5.1 \%$ by adding IC to RT or CCRT, which had no statistical significance. Also observed were significant improvement of DC by adding IC to RT or CCRT, and significant improvement of $\mathrm{LRC}$ by adding $A C$ to RT or CCRT, respectively. $\mathrm{Yu}$ et al. [9] investigated the role of IC and $A C$ 
in addition to CCRT through Bayesian NMA, based on 2,626 patients from 11 RCTs. They reported that the additional IC contributed to marginal DC benefit, but no OS difference was proved by adding either IC or AC to CCRT. Since there has been no RCT that directly compared IC + CCRT and CCRT + AC, Liu et al. [10] tried indirect comparison of these two sequences through NMA. They collected 12 RCTs in which 3,248 patients were randomly assigned to one of four treatment options: IC + CCRT, CCRT + AC, CCRT, or RT alone. They found that IC + CCRT significantly improved OS, DC, and LRC, when compared with CCRT alone, but there was no significant difference in 3 clinical outcomes between IC + CCRT and CCRT + AC or between CCRT $+A C$ and CCRT. Based on these observations, they suggested that IC + CCRT seem more promising. The update of the MACNPC meta-analysis, based on 4,806 locoregionally advanced nasopharynx cancer patients from 19 RCTs was published in 2015 by Blanchard et al. [1]. They reported that there was significant OS benefit by CCRT, which became more prominent by adding AC to CCRT. This publication was the first one that reported the results in favor of $A C$ with respect to $0 S$. The same conclusion was elucidated IPD meta-analysis published in 2017 by Ribassin-Majed et al. [11], which included 5,144 patients from 20 RCTs.

\section{Discussion}

Several recent and important meta-analysis reports on the role of additional chemotherapy to CCRT have been briefly reviewed. Many of the included trials were repeatedly analyzed by the different authors of these meta-analysis. However, no solid and concrete conclusions could be drawn. This may be because of the heterogeneity of the included trials' quality, the improper statistical methods, and/or the authors' biases which might have been based on their institutional preference. In fact, a few meta-analysis publications actually seemed to have focused on the preferred way of combination at their own institutes.

In addition to the rationale of improving $D C, I C$ has been applied mainly to reduce the initial tumor burden before the definitive local therapy is applied. In other words, less aggressive local therapy could be considered if favorable response to IC is achieved. However, IC has a few practical pitfalls in this aspect. First, though the overall response rate by the up-to-date systemic chemotherapy is expected to be $75 \%$ or even higher, still significant proportion of the patients do not achieve favorable response following IC, who subsequently may have lower chance of cure than upfront CCRT. Second,
IC can frequently compromise the patients' general condition which often necessitates the modification of the subsequent treatment schemes: CCRT with lower chemotherapy dose, omission of chemotherapy cycles during RT, or lower RT dose than initially planned, respectively. Though not all publications mentioned the data on the risk of side effect issues, the clinical benefit by IC (and AC) practically should have been achieved with the cost of more frequent and more severe acute and chronic side effects. Third, which seems the most important one to the radiation oncologists, the interpretation of post-IC imaging becomes very difficult from time to time. The target delineation for the RT planning not infrequently becomes confounded by the unpredicted, exaggerated, and extraordinary changes on the post-IC imaging studies. Should the initial gross tumor lesions did not shrink concentrically, it is reasonable to delineate the target volume with the reference to the initial imaging study, in order not to compromise the local tumor control probability. This can lead to unsuccessful and undesirable target volume delineation, through which the lessening of RT-related morbidities cannot be expected in turn.

Based on the current review, the major clinical benefit that can be expected by the addition of IC seems improved DC, but improved OS was not consistent. Though not in RCT or meta-analysis format, the role of IC was investigated based on the Korean multi-institutional data (KROG 11-06 study) using the propensity score matching (PSM) analysis [13]. After matching 150 patients' pairs, no differences in DC, PFS, and OS were observed, while the omission of IC (CCRT alone) was significantly favorable with respect to LRC. This report, even though with rather small patients' number, is quite imperative as it was based on Korean data. The role of AC seems more difficult to interpret based on the inconsistent conclusions through this review. The similar PSM analysis in an effort to investigate the role of $A C$ following CCRT was done based on Korean multi-institutional data (KROG 11-06 study) by Ahn and Kim [14], and after matching 239 patients' pairs, AC contributed to the improved DC without improving OS or LRC.

At author's institute, upfront CCRT alone has long been the main principle in treating the patients with locoregionally advanced nasopharynx cancer. From 2006 till 2013, the clinical outcomes of 159 nasopharynx cancer patients were analyzed and the 5-year OS rate of 89.6\% was achieved [15]. Among all patients, $85 \%$ received CCRT, none received IC and about onethird received $A C$ in addition to CCRT, however, the addition of $A C$ exerted favorable contribution to no clinical outcomes at all. I would speculate, based on our own data, that this high enough OS outcome could not have been further improved 
even though IC or AC had been added to CCRT routinely. Instead, the additional chemotherapy, however, could have increased the risk of acute and chronic side effects.

Notwithstanding the pros and cons of IC, however, I would consider adding IC before RT or CCRT in occasional encountered peculiar clinical setting, when the tumor is very bulky, and located just adjacent to the critical normal structures, such as the brain, the brainstem, or the optic apparatus. If a favorable response following IC is achieved, the subsequent reduction of RT target volume would become feasible, and subsequently less severe RT-related adverse effects could be expected.

By nature, all medical practices have the potential clinical benefits, and, at the same time, the potential detrimental effects. Both the addition of IC and/or AC is to be naturally associated with the increased risk of adverse effects and subsequent worsening of life quality of the patients. And, at the same time, the increased treatment cost and duration is to be inevitably incurred. In medical practice, the issues of cost, effectiveness, and value should be carefully evaluated $[16,17]$. The value should be put more on the patients' side than on the healthcare providers' side. The value should always include the clinical outcomes, which usually include survival outcomes and quality of life issue. In addition, all should keep on the line of cost-effectiveness ratio. It is natural that the addition of IC or $A C$ to CCRT can lead to not only the increased risk of systemic toxicity, but also the increased overall treatment cost and duration. Even though the immediate clinical outcomes, which typically include $L R C, D C$, and $O S$, become apparently better following the addition of IC or AC, once the unwanted severe toxicity is encountered, the patients should suffer from the lifelong hampered quality of life needing the additional effort and cost for recovery and rehabilitation. Therefore, their use should be carefully determined based on the potential benefits in the major clinical outcomes (OS, PFS, LRC, and DC) and the potential detrimental effects to the patients (adverse effects, quality of life, and treatment duration and cost). Almost all reports in this review, except a few, failed to document the toxicity and life quality issues, not to mention about the treatment cost and duration issues. If the clinical benefits are not big enough in relation with the detriments, omission of the additional IC or AC seems a wise choice.

In the architectural society, there has been a famous and common motto of 'less is more' which means 'minimalism'. The idea of freedom and essence of living can become better transmitted by the simpler design. Likewise, in the oncologic practices, 'less may be more' as well. Instead of more treatment that frequently leads to more severe, more frequent, and longer-term toxicities, less but effective treatment may be the good enough option to be chosen. This is even more reasonable if some promising and descent salvage modality is available even following treatment failure. Locoregional recurrence following high dose RT, in most cases, could be manageable by high dose re-irradiation employing either intensity-modulated RT or particle beam therapy, which usually can lead to the reasonably high salvage rate.

\section{Conclusion}

Other than the proven advantage of CCRT over RT alone, there seems to be no concrete evidences in favor of routine addition of IC or AC to CCRT in managing the patients with locoregionally advanced nasopharynx cancer, based on the heterogeneous conclusions reported by 11 meta-analysis publications. The issues of cost-effectiveness and value should be carefully evaluated in determining the actual treatment sequence. 'Less is more', and CCRT alone should be the current standard for the locoregionally advanced nasopharynx cancer patients.

\section{Conflict of Interest}

No potential conflict of interest relevant to this article was reported.

\section{References}

1. Blanchard $P$, Lee $A$, Marguet $S$, et al. Chemotherapy and radiotherapy in nasopharyngeal carcinoma: an update of the MAC-NPC meta-analysis. Lancet Oncol 2015;16:645-55.

2. Song $Y$, Wang $W$, Tao G, Zhou X. Survival benefit of induction chemotherapy in treatment for locally advanced nasopharyngeal carcinoma: a time-to-event meta-analysis. Oral Oncol 2015;51:764-9.

3. Tan $T H$, Soon $Y Y$, Cheo $T$, et al. Induction chemotherapy for locally advanced nasopharyngeal carcinoma treated with concurrent chemoradiation: a systematic review and metaanalysis. Radiother Oncol 2018;129:10-7.

4. Wang M, Tian H, Li G, et al. Significant benefits of adding neoadjuvant chemotherapy before concurrent chemoradiotherapy for locoregionally advanced nasopharyngeal carcinoma: a meta-analysis of randomized controlled trials. Oncotarget 2016;7:48375-90.

5. Chen YP, Guo R, Liu N, et al. Efficacy of the additional 
neoadjuvant chemotherapy to concurrent chemoradiotherapy for patients with locoregionally advanced nasopharyngeal carcinoma: a Bayesian network meta-analysis of randomized controlled trials. J Cancer 2015;6:883-92.

6. Chen YP, Tang LL, Yang 0 , et al. Induction chemotherapy plus concurrent chemoradiotherapy in endemic nasopharyngeal carcinoma: individual patient data pooled analysis of four randomized trials. Clin Cancer Res 2018;24:1824-33.

7. Chen YP, Wang ZX, Chen $L$, et al. A Bayesian network metaanalysis comparing concurrent chemoradiotherapy followed by adjuvant chemotherapy, concurrent chemoradiotherapy alone and radiotherapy alone in patients with locoregionally advanced nasopharyngeal carcinoma. Ann Oncol 2015;26:20511.

8. OuYang PY, Xie C, Mao YP, et al. Significant efficacies of neoadjuvant and adjuvant chemotherapy for nasopharyngeal carcinoma by meta-analysis of published literature-based randomized, controlled trials. Ann Oncol 2013;24:2136-46.

9. Yu H, Gu D, He X, Gao X, Bian X. The role of induction and adjuvant chemotherapy in combination with concurrent chemoradiotherapy for nasopharyngeal cancer: a Bayesian network meta-analysis of published randomized controlled trials. Onco Targets Ther 2016;9:159-70.

10. Liu M, You W, Song YB, et al. The changing role of chemotherapy in locoregionally advanced nasopharyngeal carcinoma: a updated systemic review and network meta- analysis. Front Oncol 2018;8:597.

11. Ribassin-Majed L, Marguet S, Lee AWM, et al. What is the best treatment of locally advanced nasopharyngeal carcinoma? An individual patient data network meta-analysis. J Clin Oncol 2017;35:498-505.

12. National Comprehensive Cancer Network. NCCN Clinical Practice Guidelines in Oncology: head and neck cancers (Version 1.2019) [Internet]. Plymouth Meeting, PA: National Comprehensive Cancer Network; c2019 [cited 2019 Jun 24]. Available from: https://www.nccn.org/professionals/physician_ gls/default.aspx.

13. Song JH, Wu HG, Keam BS, et al. The role of neoadjuvant chemotherapy in the treatment of nasopharyngeal carcinoma: a multi-institutional retrospective study (KROG 11-06) using propensity score matching analysis. Cancer Res Treat 2016;48:917-27.

14. Ahn YC, Kim YS. Korean perspectives of nasopharynx cancer management. Chin Clin Oncol 2016;5:28.

15. Lee $H_{1}$ Ahn YC, Oh D, Nam H, Noh JM, Park SY. Tumor volume reduction rate during adaptive radiation therapy as a prognosticator for nasopharyngeal cancer. Cancer Res Treat 2016;48:537-45.

16. Porter ME. What is value in health care? N Engl J Med 2010;363:2477-81.

17. Rawlins MD. Cost, effectiveness, and value: how to judge? JAMA 2016;316:1447-8. 